\title{
Bilateral variations of median nerve formation and endpoint variation of brachiales veins
}

\author{
Murat Diramali ${ }^{1}$, Rengin Kosif ${ }^{1}$, Seda Sertel Meyvaci ${ }^{1}$, Fatih Eren Daghan ${ }^{2}$
}

Diramali M, Kosif R, Meyvaci SS, et al. Bilateral variations of median nerve formation and endpoint variation of brachiales veins. Int J Anat Var. 2019; 12(4): 46-49.

According to the descriptions in the classical books, brachial plexus is formed by the ventral rami of the cervical nerves (C5, $\mathrm{C} 6, \mathrm{C} 7$, and C8) and the first thoracic nerve (T1). The reported incidence of the brachial plexus variations, which can even differ between the upper extremities of the same person, is $13 \%$ and it is most commonly encountered between the median and musculocutaneous nerves. During a routine practice on the brachial plexus of a 65-year old male cadaver, we observed that the musculocutaneous nerve pierced the coracobrachialis muscle after giving the communicating branch in both extremities.
According to the recommendation of Buch-Hansen, we decided that the communicating branch was originating from the lateral cord on the right side and from the musculocutaneous nerve on the left side. We detected a second variation after the deepening of the dissection. The medial brachial vein was terminating into the basilic vein after passing through two roots of the median nerve bilaterally. The presented anatomical variations have certain benefits regarding clinical practice. The presence of these and similar neural and vascular variations is important in respect of the possible nerve injuries, which may be caused by a piercing or cutting trauma in the upper extremity.

Key Words: Anatomical variation; Brachial plexus; Musculocutaneus nerve; Communicating branch; Basilic vein

\section{INTRODUCTION}

A ccording to the descriptions in the classical books, brachial plexus is formed by the ventral rami of the cervical nerves (C5, C6, C7, and C8) and the first thoracic nerve (T1). In other words, upper trunk C5 and C6, middle trunk $\mathrm{C} 7$ and lower trunk $\mathrm{C} 8$ and $\mathrm{T} 1$ form the brachial plexus. As a variation, it may contain fibres of $\mathrm{C} 4$ (a pre-fixed plexus) and T2 spinal nerves (a post-fixed plexus). The divisions, which are formed as a result of the splitting of the trunks in the posterior triangle of the neck, join again in the axilla and form the cords named according to the neighbourhood with the axillary artery. Thus, the lateral cord is formed by the ventral division of the upper and middle trunks; medial cord by the ventral division of the lower trunk and posterior by the posterior division [1-3]. The peripheral nerves, which are responsible for the sensorial and motor innervation of the upper extremity, originate from the brachial plexus. But the nerves, which are responsible for the sensorial innervation of the region close to the shoulder, originate from the cervical plexus. The sensorial innervation of a small region in the medial side of the arm is provided by the intercostobrachial nerve $[4,5]$.

The reported incidence of the brachial plexus variations, which can even differ between the upper extremities of the same person, is $13 \%$ and it is most commonly encountered between the median and musculocutaneous nerves [2,6-8].

The median nerve is usually formed by two separate roots originating from the lateral and middle cord and it passes through the arm without giving branches except for the branch, which innervates the brachial artery, and participates in the innervation of the forearm and the hand $[3,6,9]$.

The musculocutaneous nerve, which originates from the lateral cord, passes through the biceps brachii and brachialis muscle after piercing the coracobrachialis muscle and ends in the sensorial branch or in the lateral side of the forearm after innervating these three muscles [3].

The knowledge about the anatomical variations of the brachial plexus are essential for a correct regional anesthesia in the upper extremity, for a clear understanding about the clinical outcomes of the neural dysfunctions and for the prevention of the nerve injuries during the operations involving the axilla and humeral head $[4,8,10]$

Due to the clinical and surgical importance of the brachial plexus, we decided to introduce the variation affecting the median and musculocutaneous nerves, and the concomitant venous variation to the literature, which we observed in the Anatomy Lab of our medical school.

\section{CASE REPORT}

During a routine practice on the brachial plexus of a 65-year old male cadaver in our Anatomy Laboratory, we observed a communication extending from the lateral to the median nerve and decided to deepen the dissection.

The dissection of the axilla and the arm was carried out with an incision extending through the ventral axillary line. Following the cautious removal of the superficial and deep fasciae, the pectoral muscle was removed in order to expose the vascular and neural structures.

We observed that the musculocutaneous nerve pierced the coracobrachialis muscle after giving the communicating branch in both extremities of our cadaver. Then it innervated the three muscles in the ventral region of the arm and continued as a lateral cutaneous nerve into the forearm.

After the isolation of the tract of the musculocutaneous and median nerves, we measured with the help of a caliper the distance between the lateral root of the median nerve and the communication originating from the musculocutaneous nerve, the thickness of the lateral root and the

${ }^{1}$ Department of Anatomy, Faculty of Medicine, Bolu Abant Izzet Baysal University, TURKEY; ${ }^{2}$ Department of Anatomy, Faculty of Medicine, Bolu Abant Izzet Baysal University, Term IV, TURKEY

Correspondence: Dr. Murat Diramali, Department of Anatomy, Faculty of Medicine, Bolu Abant Izzet Baysal University, TURKEY. Telephone +905066273685; E-mail: mdiramali@yahoo.com

Received: Jul 12, 2019, Accepted: Oct 18, 2019, Published: Oct 25, 2019 org/licenses/by-nc/4.0/), which permits reuse, distribution and reproduction of the article, provided that the original work is properly cited and the reuse is restricted to noncommercial purposes. For commercial reuse, contact reprints@pulsus.com 
communicating branch and the width of the median nerve before and after the median nerve and communicating branch unite.

The second lateral root on the right side was originating from the lateral cord at a distance of $31.31 \mathrm{~mm}$ to the lateral root. The width of the proximal and distal roots was $2.15 \mathrm{~mm}$ and $4.17 \mathrm{~mm}$ respectively. The diameter of the median nerve before and after it united with this second root was $3.62 \mathrm{~mm}$ and $6.24 \mathrm{~mm}$ respectively (Figure 1 ).

On the left side, an anastomotic branch was emerging from the musculocutaneous nerve at a distance of $18.78 \mathrm{~mm}$ to the median nerve. The thicknesses of the lateral root of the median nerve and the anastomotic branch were $3.81 \mathrm{~mm}$ and $3.08 \mathrm{~mm}$ respectively. The diameter of the median nerve before it united with this anastomotic branch was $3.45 \mathrm{~mm}$ and 5.37 $\mathrm{mm}$ respectively (Figure 2 ).

According to the recommendation of Buch-Hansen, this branch should be considered as a second lateral root of the median nerve if it is thicker than the proximal nerve and as an anastomotic branch originating from the musculocutaneous nerve if it is thinner than the proximal nerve [1]. From this point of view, we decided that the communicating branch was originating from the lateral cord on the right side and from the musculocutaneous nerve on the left side.

We detected a second variation after the deepening of the dissection. In this bilateral venous variation; the medial brachial vein, which should be terminated into the basilic vein just at the distal of the level of the emergence of the median nerve, was terminating into the basilic vein after passing through two roots of the median nerve, which were joining from the lateral. However, the lateral brachial vein had a normal course on both sides.

\section{DISCUSSION}

It was reported that the brachial plexus is a variative part of the peripheral nerve system. The musculocutaneous nerve variations, which have an incidence of $6.25 \%$ according to the literature, are mainly divided into two groups: Its absence and the presence of a communication between the musculocutaneous and median nerves [3].

Its absence does not cause any functional loss in the flexion of the arm or in the sensorial innervation of the forearm due to the fibers originating from

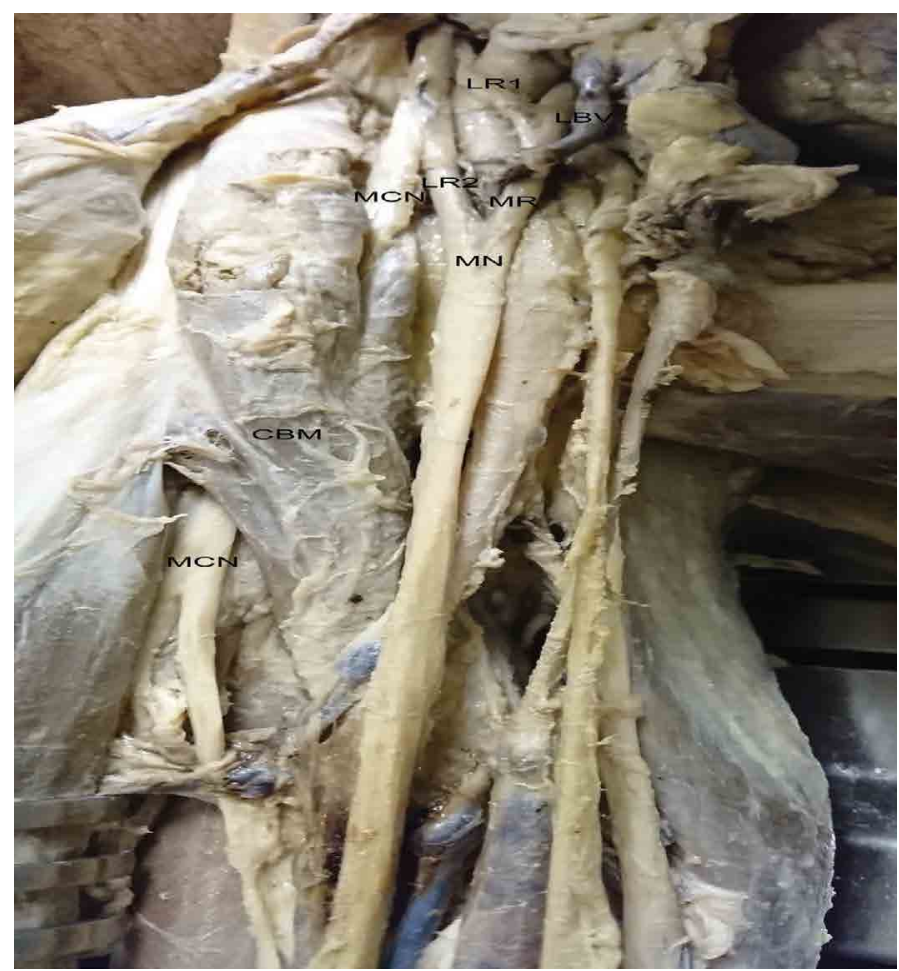

Figure 1) Musculocutaneous nerve pierces the coracobrachialis muscle after giving the communicating branch on cadaver right forearm. LR1 indicates the first lateral root of the median nerve, LR2 indicates the second lateral root of the median nerve, $M R$ indicates the medial root of the median nerve, MN indicates the median nerve, $M C N$ indicates the musculoctane nerve, LBV indicates the lateral brachial vein and CBM indicates the coracobrachialis muscle.

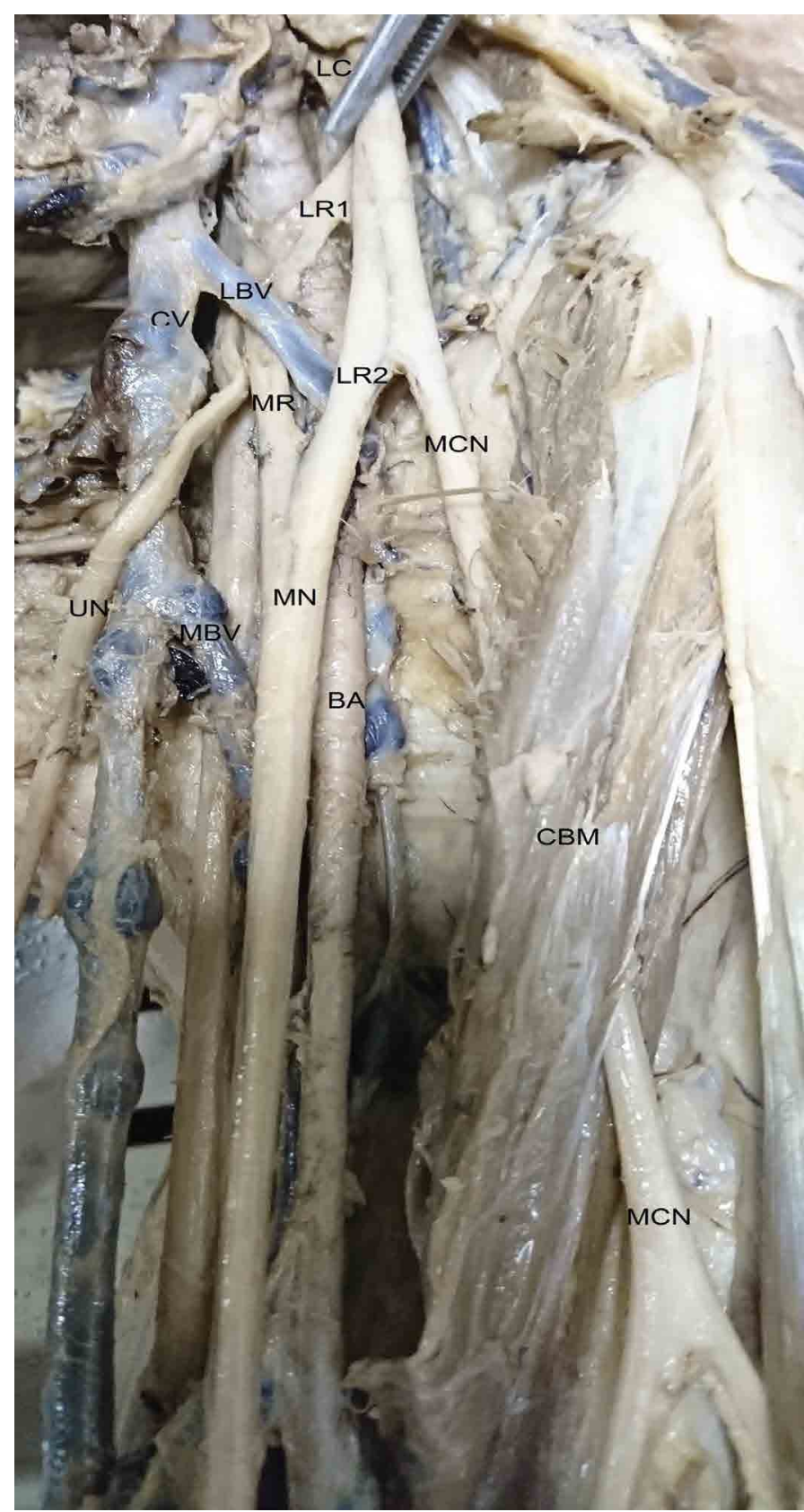

Figure 2) Musculocutaneous nerve pierces the coracobrachialis muscle after giving the communicating branch on cadaver left forearm. LR1 indicates the first lateral root of the median nerve, LR2 indicates the second lateral root of the median nerve, MR indicates the medial root of the median nerve, MN indicates the median nerve, UN indicates the ulnar nerve, MCN indicates the musculoctane nerve, LBV indicates the lateral brachial vein, $M B V$ indicates the medial brachial vein, $C V$ indicates the cephalic vein and CBM indicates the coracobrachialis muscle.

other nerves. These fibers originate mostly from the median nerve and less frequently from the lateral root of the median nerve or from the lateral cord [6].

The most common one among all brachial plexus variations is the communicating branch between the median and musculocutaneous nerves with an incidence of $1.4 \%-63.5 \%[1,11]$.

The nerve variations are important for the radiologists, anesthesiologists, and surgeons because of their clinical impacts. For example, an undetected absence of the musculocutaneous nerve may mislead a clinician, who tries to examine the results of an electromyography. Regarding the surgical interventions, the fibers of the musculocutaneous nerve may be damaged while the median nerve seems to be intact during the evaluation of the arm after a trauma or during the removal of a flap. The communicating branches affecting the musculocutaneous nerve are also important regarding the approach to the shoulder traumas and the evaluation of the nerve dysfunctions. The median nerve and its roots are close to the axillary vein, which is the upper limit in the axillary lymph node dissection, a routine procedure during the breast 
carcinoma or melanoma operations. Trap neuropathies and injuries due to the unexpected origin, course, and distribution of the nerves are common in the presence of the variant nerves [1,6,10-12].

Because of the clinical importance, the incidence of the anatomical variations should be precisely classified in order to detect them easily. Venieratos and Anagnostopoulou conducted a study on 79 human cadavers. In this study, they divided this communication, which had an incidence of $13.9 \%$, into three types according to the association with the coracobrachialis muscle. Type I: The communication was proximal to the entrance of the musculocutaneous nerve into coracobrachialis; Type II: The communication was distal to the muscle (most common); Type III: The nerve as well as the communicating branch did not pierce the muscle $[1,8]$.

Uzun et al. conducted a study on 65 neonate cadavers, which did not have any visible external malformation and reported that there was a variative junction between C4 and C5 in 20 of the 130 examined brachial plexuses and a variative junction between the medial root of the median nerve and middle trunk in 14 of this 130 brachial plexuses. They also added that these variations were not affected by the gender and body side [13].

During the dissection of a 60-year old male cadaver, Sarıkçığlu et al. observed variations affecting both the nerve system and the circulatory system. They reported that the arterial variations were on the right side and there was a superficial brachial artery below the pectoralis minor muscle originating from the axillary artery. They also stated that there was a communicating branch between the musculocutaneous and median nerves on the left side of the same cadaver and an accessory nerve originating from the lateral cord innervated the coracobrachialis muscle [14].

Fazan et al. investigated on 27 cadavers the effect of the gender, ethnic differences, and right-left sides on the incidence of the variations and found out that there was no significant difference between these parameters. The brachial plexus received branches from C5 only in 13 of the investigated 54 brachial plexuses and only in 3 cases, there was a contribution from T2. They did not observe any variation in the origins of the axillary and musculocutaneous nerves. The musculocutaneous nerve gave a communicating branch to the median nerve after piercing the coracobrachialis muscle in four cadavers. They also reported that the median nerve constituted of two lateral and one median roots in 28 cadavers. In 24 of these 28 median nerves, both lateral roots originated from the lateral cord while in the remaining 4 median nerves, one of the lateral roots emerged from the ventral division of the middle trunk and the second one originated from the lateral cord [10].

During a routine dissection of a 50-year old cadaver, Kocabiyık et al. observed an accessory branch, which united with the median nerve after leaving the musculocutaneous nerve in the middle of the arm and passing through the biceps brachii and brachialis muscle. In spite of the presence of this accessory branch, the biceps brachii and the brachialis muscle were innervated by the musculocutaneous nerve and this nerve continued through the forearm as the lateral antebrachial cutaneous nerve [15].

Sevinc et al. observed a variation affecting the formation of the median nerve during the dissection of a 55 -year old male cadaver. The lateral root of the median nerve was divided into proximal and distal branches after leaving the lateral cord. The proximal branch formed the median nerve after joining the medial root of the median nerve while the distal branch joined the median nerve. They have also added that they observed a communication connecting the musculocutaneous and median nerves in the same cadaver [9].

During the routine anatomical dissection of a male cadaver. Fregnani et al. determined that the lateral cord continued as the lateral root of the median nerve without giving the musculocutaneous nerve and all three muscles of the arm were innervated by the median nerve and that the only branch originating from the lateral fascicle was the lateral pectoral nerve. The cadaver did not have any other nervous system variation in the upper extremity, but the cephalic vein was terminated into the jugular vein instead of the axillary vein [6].

Sinha et al. conducted a study on 40 upper extremities and detected 5 different variations: two upper subscapular nerves originating together with the lower subscapular nerve from the upper trunk $(n=1)$; axillary and upper subscapular nerves originating from the upper trunk $(n=1)$; the median nerve was comprised of three roots - two from the lateral cord and one from the medial cord $(n=1)$; the ulnar nerve was formed by two roots $(n=1)$; a communicating branch extending from the musculocutaneous nerve to the median nerve $[1,11]$

Claassen et al. investigated 167 upper extremities and detected brachial plexus variations in 60 extremities. They reported that the variations were observed most frequently in the median nerve (46 cases) and in the musculocutaneous nerve (13 cases). They also added that the most common variations were as follows: the musculocutaneous nerve did not pierce the coracobrachialis muscle, the nerve had two roots and gave fibers to the median nerve [7].

During a routine anatomical dissection, Nascimento et al. determined a variation in the upper extremity of a male cadaver: First, as usual, the musculocutaneous nerve originated from the lateral cord and then pierced the coracobrachialis muscle and formed the lateral antebrachial cutaneous nerve together with the motor branches of the biceps brachii and brachialis muscles. But then it united with the median nerve in the middle of the arm [16].

Nasrabadi et al. dissected an Iranian male cadaver and observed an unexpectedly thin musculocutaneous nerve, which originated from the lateral cord before piercing the muscle on the ventral face of the coracobrachialis muscle and gave a communicating branch to the median nerve [3].

Gelmi et al. observed a communicating branch during a routine anatomical dissection, which was extending from the musculocutaneous nerve to the median nerve after passing through the coracobrachialis muscle. In the same upper extremity, the biceps brachii was atrophic although there was no variation related to the innervation of the muscle by the musculocutaneous nerve [8].

In our case, the communicating branch united with the median nerve before piercing the coracobrachialis muscle like in the observations of Sevinç et al. and Gelmi et al. This variation was also consistent with the Type I described by Vanietratos and Anagnostopoulou. The only difference of our variation from Type I was the emergence of the communicating branch from the lateral cord in the right side. Unlike Gelmi et al., we did not observe any atrophy in the muscles of the upper extremity. Similar to the variation reported by Sarıcçığlu et al., we observed a vascular variation in our cadaver. However, this variation was related to the veins in our case. The medial brachialis vein, which should be normally terminated into the basilic vein at more distal levels, terminated into the basilic vein at a more proximal level after passing through two roots of the median nerve on both sides.

In the $5^{\text {th }}$ embryonic week, the arm muscles start to develop from the myotome and brachial plexus starts to develop from the spinal nerves originating from the neural tube. The brachial plexus reaches its final shape in the $8^{\text {th }}$ week. During this process, the axons of the spinal nerves are divided to form the ventral and dorsal divisions after extending to the distal to reach the extremity buds. The median and ulnar nerves emerge before the ventral division and then the musculocutaneous nerve emerges from the median nerve. The migration of the axons is regulated by the expression of the chemoattractants and chemorepulsants specific to a certain region and it was suggested that any change in this signalization might lead to variations $[3,11]$.

\section{CONCLUSION}

The presented anatomical variations have certain benefits regarding clinical practice. The presence of these and similar neural and vascular variations is important in respect of the possible nerve injuries, which may be caused by a piercing or cutting trauma in the upper extremity, and they also enable to explain the symptoms except the known symptoms encountered in the flexor muscles of the elbow or in the sensorial function of the forearm and hand.

\section{REFERENCES}

1. Venieratos D, Anagnostopoulou S. Classification of Communications between the Musculocutaneous and Median Nerves. Clin Anat. 1998;11:327-31.

2. Gregory J, Cowey A, Jones $\mathrm{M}$, et al. The Anatomy, Investigations and Management of Adult Brachial Plexus Injuries. Orthop Trauma. 2009;23:420-32.

3. Nasrabadi HT, Abedelahi A, Shoorei H, et al. A Variation of Musculocutaneous Nerve without Piercing the Coracobrachialis Muscle While Communicating to the Median Nerve: A Case Report and Literature Review. Int J Surg Case Rep. 2017;41:453-55. 
4. Bollini CA, Wikinski JA. Anatomical Review of Brachial Plexus. Tech Reg Anesth Pain Med. 2006;10:69-78.

5. Christophe JL, Berthier F, Boillot A, et al. Assessment of Topographic Brachial Plexus Nerves Variations at the Axilla Using Ultrasonography. Br J Anaes. 2009;103:606-12.

6. Fregnani JH, Macéa MI, Pereira CS, et al. Absence of the Musculocutaneous Nerve: A Rare Anatomical Variation with Possible Clinical-Surgical Implications. Sao Paulo Med J. 2008;126:288-90.

7. Claassen H, Schmitt O, Wree A, et al. Variations in Brachial Plexus with Respect to Concomitant Accompanying Aberrant Arm Arteries. Ann Anat. 2016;208:40-8

8. Gelmi CAE, Pedrini FA, Matteo F, et al. Communication Between Median and Musculocutaneous Nerve at the Level of Cubital Fossa - A Case Report. Transl Res Anat. 2018;11:1-4.

9. Sevinc O, Arifoglu Y, Barut C, et al. Variation in the Formation of the Median Nerve Involving A Communicating Branch from the Musculocutaneous Nerve. Case Rep Clin Pract Rev. 2007; 8:143-6.
10. Fazan VPS, Amadeu AS, Caleffi AL, et al. Brachial Plexus Variations in Its Formation and Main Branches. Acta Cir Bras. 2003;18:14-18.

11. Sinha RS, Chaware PN, Pandit SV, et al. Variations in the Branching Pattern of Brachial Plexus with Their Embryological and Clinical Correlation. J Morphol Sci. 2012;29:167-70.

12. Hayashi M, Shionoya K, Hayashi S, et al. A Novel Classification of Musculocutaneous Nerve Variations: The Relationship Between the Communicating Branch and Transposed Innervation of the Brachial Flexors to the Median Nerve. Ann Anat. 2017;209:45-50.

13. Uzun A, Bilgic S. Some Variations in the Formation of the Brachial Plexus in Infants. Tr J of Med Sci. 1999;29:573-77.

14. Sarkecioglu L, Coskun N, Ozkan O. A Case with Multiple Anomalies in the Upper Limb. Surg Radiol Anat. 2000;23:65-8.

15. Kocabiyık N, Yalcın B, Yazar F, et al. An Accessory Branch of Musculocutaneous Nerve Joining Median Nerve. Neuro Anat. 2005;4:13-15.

16. Nascimento SRR, Ruiz CR, Pereira E, et al. Rare Anatomical Variation of the Musculocutaneous Nerve - Case Report. Rev Bras Ortop. 2016;51:366-369. 